\title{
A Note on Series Parallel Irreducibility
}

\author{
by \\ BERNARD P. ZEIGLER* \\ Department of Computer and Communication Sciences \\ The University of Michigan \\ Ann Arbor, Michigan 48104
}

ABSTRACT

\begin{abstract}
A new criterion for series-parallel irreducibility is given which makes no reference to underlying semigroups but involves only series-parallel connection operations.
\end{abstract}

A semi-automaton or transition system is a triple $\langle X, Q, M\rangle$ where $X, Q$ are finite sets (of input symbols and internal states respectively), and $M: Q \times X \rightarrow Q$ is the transition function. (In the usual abuse of notation we write $M$ for $\langle X, Q$, $M>$.) In this note we shall characterize the semi-automata which are irreducible with respect to series-parallel decomposition. This augments the definition of Krohn and Rhodes [1] (see also Arbib's formulation in [2]), which in an essential way required the specification of output maps and thus held only for full automata, i.e., machines of the form $\langle S, Q, O, M, N\rangle$, where $O$ is the outut set and $N$ : $Q \rightarrow O$, the output function. Moreover, their definition of irreducibility for machines made direct reference to semigroups while the definition we shall give makes reference only to series-parallel connection operations. Except for changes in notation the presentation follows that of [2] (Chapters 3 and 5).

Let $S(M)$ denote the semigroup of $M$, i.e.,

$$
S(M)=\left\{\tilde{M}(, x): Q \rightarrow Q \mid x \in X^{*}\right\},
$$

where $\tilde{M}$ is $M$ extended to $X^{*}$. Given a semigroup $S$, let $M_{S}$ denote the semigroup transition system, i.e., $M_{S}: S^{1} \times S \rightarrow S^{1}$ with $M_{S}(1, s)=s$ and $M_{S}\left(s, s^{\prime}\right)=s s^{\prime}$ for all $s, s^{\prime} \in S$. Note that $S\left(M_{S}\right)=S .^{1}$

In the following we consider as usual only connected machines with specified starting state.

Given transition functions $M_{i}: Q_{i} \times X_{i} \rightarrow Q_{i}, i=1$, 2, we say that $M_{2}$ divides $M_{1}$ (written $M_{2} \mid M_{1}$ ) if there exist $Q_{1}^{\prime} \subseteq Q_{1}$ and maps $g: X_{2} \rightarrow X_{1}^{*}$, $h: Q_{1}^{\prime} \rightarrow Q_{2}$ (onto) such that

(1) $Q_{1}^{\prime}$ is closed under $g\left(X_{2}\right)^{*}$ and

(2) for all $q_{1} \in Q_{1}^{\prime}, s \in X_{2}, h\left(\tilde{M}_{1}\left(q_{1}, g(s)\right)=M_{2}\left(h\left(q_{1}\right), s\right)\right.$.

* Research was sponsored by National Institutes of Health, Grant No. GM-12236-03; Office of Naval Research, Contract No. N00014-67-A-0181-011; and U.S. Army Research Office (Durham), Grant No. DA-31-124-ARO-D-483.

$1 S^{1}$ is the smallest monoid containing $S$. 
Given $\langle X, Q, M\rangle$ and a positive integer $n$ define $\Pi^{n} M=\left\langle X, Q^{n}, \Pi^{n} M\right\rangle$ by $\Pi^{n} M\left(q_{1}, \cdots, q_{n}, s\right)=\left(M\left(q_{1}, s\right), \cdots, M\left(q_{n}, s\right)\right)$ for all $\left(q_{1}, \cdots, q_{n}, s\right) \in Q^{n} \times X$. $\Pi^{n} M$ represents $n$ copies of machine $M$ (possibly in different states) which are run in parallel and are fed the same input symbol.

Definition. $M_{2}$-divides $M_{1}\left(\left.M_{2}\right|_{\pi} M_{1}\right)$ if there is a positive integer $n$ such that $M_{2} \mid \Pi^{n} M_{1}$. We remark that division, and $\pi$-division are transitive relations.

$M_{2}$ mutually $\pi$-divides $M_{1}\left(M_{2} \equiv_{\pi} M_{1}\right)$ if $\left.M_{2}\right|_{\pi} M_{1}$ and $\left.M_{1}\right|_{\pi} M_{2}$. We require the following statements.

(1) $M_{2} \mid M_{1}$ implies $S\left(M_{2}\right) \mid S\left(M_{1}\right) .^{2}$

(2) $S\left(M_{2}\right) \mid S\left(M_{1}\right)$ implies $M_{2} \mid M_{S\left(M_{1}\right)}$.

(3) $\left.M_{S(M)}\right|_{\pi} M$.

(4) $S\left(\Pi^{n} M\right)=S(M)$.

Proofs may be found in Chapter 1 of [4]. Suffice it to say that (1) and (2) are well-known; (3) is a slight extension of Fact 2.14b, Chapter 5 of [3]. For (4) we note that

$$
\widetilde{\Pi}^{n} M\left(q_{1}, \cdots, q_{n}, x\right)=\left(\tilde{M}\left(q_{1}, x\right), \cdots, \tilde{M}\left(q_{n}, x\right)\right),
$$

and examining the Myhill equivalences relations, we have

$$
\begin{aligned}
x \equiv_{\Pi^{n} M} y & \Leftrightarrow \text { for all }\left(q_{1}, q_{2}, \cdots, q_{n}\right) \in Q^{n}, \widetilde{\Pi}^{n} M\left(q_{1}, \cdots, q_{n}, x\right)= \\
& \widetilde{\Pi}^{n} M\left(q_{1}, \cdots, q_{n}, y\right) \\
& \Leftrightarrow \text { for all } q \in Q, \tilde{M}(q, x)=\tilde{M}(q, y) \\
& \Leftrightarrow x \equiv_{M} y .
\end{aligned}
$$

Hence $S\left(\Pi^{n} M\right)=X^{*}\left|\equiv_{\Pi^{n} M}=X^{*}\right| \equiv_{M}=S(M)$.

PROPOSITION 1. $S\left(M_{2}\right) \mid S\left(M_{1}\right)$ if and only if $\left.M_{2}\right|_{\pi} M_{1}$.

Proof. Assume that $S\left(M_{2}\right) \mid S\left(M_{1}\right)$. Then from (2), $M_{2} \mid M_{S\left(M_{1}\right)}$. Also from (3) $\left.M_{S\left(M_{1}\right)}\right|_{\pi} M_{1}$ so by transitivity $\left.M_{2}\right|_{\pi} M_{1}$.

Conversely, assume that $\left.M_{2}\right|_{n} M_{1}$. Then for some $n, M_{2} \mid \Pi^{n} M_{1}$ so by (1) $S\left(M_{2}\right) \mid S\left(\Pi^{n} M_{1}\right)$. Recognizing that $S\left(\Pi^{n} M_{1}\right)=S\left(M_{1}\right)$ from (4) completes the proof.

We see that Proposition 1 allows re-interpretation of semigroup division in terms of $\pi$-division. This is not true for ordinary division; to make the converse of (1) hold, output maps have to be added to the semigroups as in Theorem 7.3.10 of [2]. The best that we can get from (1) and (2) is

(5) $S\left(M_{2}\right) \mid S\left(M_{1}\right)$ if and only if $M_{2} \mid M_{S\left(M_{1}\right)}$. An interesting consequence of Proposition 1 is

COROLLARY 2. $M_{1} \equiv_{\pi} M_{2}$ if and only if $S\left(M_{1}\right) \cong S\left(M_{2}\right)$.

Proof. Apply Proposition 1 twice.

The standard definitions of irreducibility are:

(a) A semigroup $S$ is irreducible if whenever $S \mid S_{2} \times_{Z} S_{1}$ then $S \mid S_{2}$ or $S \mid S_{1}$. (Here $S_{2} \times{ }_{Z} S_{1}$ is a semidirect product of $S_{1}$ by $S_{2}$ with connecting map $Z$.)

2For semigroups $S_{i}, i=1,2, S_{1} \mid S_{2}$ if $S_{1}$ is a homorphic image of sub-semigroup of $S_{2}$. 
(b) A machine $N^{*} \quad$ irreducible if whenever $M \mid M_{2} \times{ }_{\mathrm{Z}} M_{1}$ then $M \mid M_{2}$ or $M \mid M_{1}$. (Here $M_{2} \times_{2}: I_{1}$ is the series-parallel cascade of $M_{1}$ followed by $M_{2}$ with connecting map $Z$.)

(c) A machine $M$ is s-irreducible if whenever $M \mid M_{2} \times_{Z} M_{1}$ then $M \mid M_{S\left(M_{2}\right)}$ or $M \mid M_{S\left(M_{1}\right)}$.

We add the definition:

(d) A machine $M$ is $\pi$-irreducible if whenever $M \mid M_{2} \times_{Z} M_{1}$ then $\left.M\right|_{\pi} M_{2}$ or $\left.M\right|_{\pi} M_{1}$.

Theorems 8.3.6 and 8.3.7 ([2], p. 4) state that $M$ is $s$-irreducible if and only if $S(M)$ is irreducible. On the other hand, while $M$ is irreducible implies $S(M)$ is irreducible, the converse does not hold. ${ }^{3}$ Using on Proposition 1 we can now show that the equivalence does hold for $\pi$-irreducibility.

THEOREM 3. $M$ is $\pi$-irreducible if and only if $M$ is s-irreducible.

Proof. $M$ is $\pi$-irreducible $\Leftrightarrow$ if $M \mid M_{2} \times{ }_{2} M_{1}$ then $\left.M\right|_{\pi} M_{2}$ or $\left.M\right|_{\pi} M_{1} \Leftrightarrow$ if $M \mid M_{2} \times_{Z} M_{1}$ then $S(M) \mid S\left(M_{2}\right)$ or $S(M) \mid S\left(M_{1}\right)$ (from Proposition 1) $\Leftrightarrow$ if $M \mid M_{2} \times_{Z} M_{1}$ then $M \mid M_{S\left(M_{2}\right)}$ or $M \mid M_{S\left(M_{1}\right)}$ (from [5]) $\Leftrightarrow M$ is s-irreducible.

In conclusion, we have seen that the irreducibles are strictly included in the $s$-irreducibles which are co-extensive with the $\pi$-irreducibles. What this says is that although a machine $M$ which is $s$-irreducible but not irreducible has a seriesparallel decomposition into machines $M_{1}, M_{2}$ such that neither $M_{1}$ nor $M_{2}$ can simulate $M$, still it must be that by taking a suitable number of copies of either $M_{1}$ or $M_{2}$ we can simulate $M$, i.e., $\left.M\right|_{\pi} M_{1}$ of $\left.M\right|_{\pi} M_{2}$. Finally we note that Theorem 3 enables us to relate the $s$-irreducible machines given by the Krohn-Rhodes theory (the simple group and unit actions) entirely to machine decomposition operations without reference to semigroup concepts.

Added in proof: A related paper was presented at the Eleventh Annual Symposium on Switching and Automata Theory, Santa Monica, California.

\section{REFERENCES}

[1] K. B. KroHn and J. L. Rhodes, "Algebraic Theory of Machines", Mathematical Theory of Automata, p. 371, Polytechnic Press, 1963.

[2] R. E. Kalman, P. L. FAlb and M. A. Arbib, Topics in Mathematical Systems Theory, Chapters 7-8, McGraw-Hill, 1969.

[3] M. A. ArbiB, Algebraic Theory of Machines, Languages and Semi-Groups, pp. 41-46, Academic Press, 1968.

[4] B. P. ZeIGler, "On the Feedback Complexity of Automata," University of Michigan Technical Report No. 08226-6-T, Ph.D. Dissertation, Computer and Communication Sciences Department, Ann Arbor, Michigan, 1968.

(Received 20 August 1969)

${ }^{3}$ Actually, these are proved for full machines but can easily be shown to be true for semiautomata. 